\title{
Effect of Glutathione on Lung Activator Protein-1 Activation and Heme Oxygenase-1 Induction in the Immature Rat
}

\author{
GUANG YANG, MYCHELLE L. SHEGOG, AND PHYLLIS A. DENNERY \\ Department of Pediatrics, Stanford University School of Medicine, Stanford, California, U.S.A.
}

\begin{abstract}
ABS
Differences in lung heme oxygenase-1 (HO-1) regulation
have been demonstrated in newborn $(<12$ h old) and adult $(>2$
month old) rats after exposure to hyperoxia. Contrary to adults,
neonates do not demonstrate increased lung HO-1 induction nor
transcription factor activator protein-1 (AP-1) binding in hyper-
oxia. Because AP-1 activation can be posttranslationally modi-
fied by oxidants or reductants, we investigated whether differ-
ences in lung glutathione (GSH) content account for the
maturational differences in AP-1 activation and subsequent HO-1
gene regulation after hyperoxia. Neonatal rats were injected with
either 1-buthionine-[S, R] sulfoximine (BSO), diamide, or selen-
ite during the 72-h hyperoxic exposure. Lung GSH content,
glutathione disulfide (GSSG) content, AP-1 binding, and HO-1
mRNA were evaluated. The ratios of GSSG to GSH were used to
\end{abstract}
reflect the GSH redox state in the lungs. Changes in lung GSSG/GSH ratio did not alter AP-1 binding but did increase HO-1 mRNA in neonates. These data suggest that the neonatal lung is relatively resistant to AP-1 activation and HO-1 induction by GSH perturbation. (Pediatr Res 52: 34-39, 2002)
Abbrevi
BSO, buthionine sulfoximine
HO-1, heme oxygenase-1
AP-1, activator protein-1
GSH, glutathione
GSSG, glutathione disulfide
TGSH, total glutathione

$\mathrm{HO}-1$, the rate-limiting enzyme in the degradation of heme to bilirubin, is a stress response protein with antioxidant properties. This enzyme is differentially expressed in the lungs of neonatal and adult rats after exposure to hyperoxia. Contrary to adult rats, neonates did not demonstrate increased HO-1 induction (1) nor lung transcription factor AP-1 binding (2) in hyperoxia. Nonetheless, the neonates were able to compensate for the lack of HO-1 mRNA induction by elevating lung HO-1 protein and total HO activity, perhaps through posttranscriptional mechanisms. It is not clear what events mediate the lack of HO-1 mRNA induction and the lack of change in AP-1 binding in the neonatal lungs after hyperoxia.

The transcription factor AP-1 is a known stress response complex that functions in signal transduction by inducing the expression of specific downstream genes. The AP-1 complex consists of various heterodimers of fos/jun proteins or homodimers of jun/jun proteins. Several mechanisms are believed to be involved in regulating AP-1 transcriptional activ-

Received December 4, 2000; accepted March 8, 2002.

Correspondence: Phyllis A. Dennery, M.D., Department of Pediatrics, Stanford University School of Medicine, 750 Welch Rd. \#315, Palo Alto, CA 94304, U.S.A.; e-mail: dennery@leland.stanford.edu

Supported by National Institutes of Health grants HL-52701 and HL-58752 (P.A.D.), an ALA Career Investigator Award (P.A.D.), and the Mary L. Johnson and Hess Funds of Stanford University. ity, including differential gene expression of fos or jun, conformational alterations, posttranslational modification, and altered DNA-binding specificities of the heterodimers (3). In vitro studies demonstrate that AP-1 DNA-binding activity can be modulated by oxidants or reductants via a conserved cysteine residue on the DNA binding domains of either jun or fos protein (4). Alterations of AP-1 binding activity are also observed in many in vivo situations where the cellular GSH content is changed, such as in oxidative stress.

GSH coexists with its disulfide, GSSG. Either increased GSSG content or an increased GSSG/GSH ratio can reflect cellular oxidative stress. Many studies have shown that antioxidant agents $(5,6)$ or antioxidant enzyme systems such as the thioredoxin/thioredoxin reductase complex (7-9) decrease the ratio of GSSG/GSH and enhance AP-1 binding activity, implying that AP-1 activation is favorable in a more reduced cellular environment. However, an increased ratio of GSSG/ GSH has also been shown to enhance transactivation of AP-1 (7). GSH depletion induces HO-1 mRNA in rat brains (10). Could alterations in lung GSH content account for the lack of increase in HO-1 mRNA in the immature lung via AP-1?

There are several AP-1 encoding elements in the enhancer regions of the mouse HO-1 promoter, and these are important in metal- and heme-induced HO-1 expression (11-14). Hyper- 
oxia-induced HO-1 expression is also associated with enhanced AP-1 binding in adult rat lungs $(2,15)$ but not in neonates (2). We investigated the role of lung GSH content in modulating AP-1 DNA-binding activity and HO-1 mRNA in the neonatal and adult rat exposed to hyperoxia and determined whether altering GSH content or GSSG/GSH ratio in the neonate modified AP-1 binding.

\section{MATERIALS AND METHODS}

\section{Animal Models}

Litters of Wistar rat pups (D0, $<12 \mathrm{~h}$ old) with their mothers, and 2-mo-old adult male rats (adults, $>2$ mo old) were obtained from Simonsen Labs (Gilroy, CA, U.S.A.). The animals were kept in a 12-h light-dark cycle and allowed to feed ad libitum until the time of experimentation.

All animal protocols were reviewed and approved by the Administrative Panel on Laboratory Animal Facility at Stanford University.

\section{Experimental Design}

The D0 or adult rats were placed in a flow-through, airtight Plexiglas cylindrical exposure chamber (Vreman Scientifics, Los Altos, CA, U.S.A.) that had ports for gas entry and exit, and exposed to either air or hyperoxia $\left(>95 \% \mathrm{O}_{2}\right)$ as described previously (1). One hour before exposure, some of the D0 rats were injected i.p. with diamide, $25 \mathrm{mg} / \mathrm{kg}$, a dose that is known to increase HO-1 mRNA in adult rats (16). In other instances, $1 \mathrm{~h}$ before exposure, selenite was injected to the D0 rats at 5 $\mu \mathrm{mol} / \mathrm{kg}$, a dose shown to increase GSH levels (17). Additionally, $1 \mathrm{~h}$ before exposure, some neonatal rats received BSO, 3 $\mathrm{mmol} / \mathrm{kg}$, a dose known to deplete cellular GSH content in vivo (18). All injections were performed at 12-h intervals for the 72-h period of hyperoxia. Controls were similarly injected with saline. Lastly, some adult rats were similarly injected with diamide and exposed to hyperoxia to allow for comparison with the D0 rats. After $3 \mathrm{~d}$ of exposure, the pups were killed by decapitation and the adults by brief $\mathrm{CO}_{2}$ narcosis followed by decapitation. The lungs were excised and rinsed in cold PBS on ice and further perfused with cold $0.15 \mathrm{M} \mathrm{KCl}$ to remove any blood. Three neonate or two adult lung samples were pooled per experiment.

\section{Determination of HO-1 mRNA Levels}

Plasmid and probe preparation. The plasmid $\mathrm{pBKRHO} 1$ was constructed in pBluescript II SK- using a rat HO-1 cDNA fragment as previously described (1) and amplified by PCR. The housekeeping gene $\beta$-actin c-DNA (American Type Culture Collection, Manassas, VA, U.S.A.) was prepared by standard methods. Labeled probes were prepared by the random primer method using ${ }^{32} \mathrm{P}-\mathrm{dCTP}$ as described previously (1).

Northern hybridization. Lung RNA was isolated using Trizol reagent (Invitrogen, Carlsbad, CA, U.S.A.) and quantitated spectrophotometrically at $260 \mathrm{~nm}$. RNA, $20 \mu \mathrm{g}$, was electrophoresed and probed as previously described (1). For reprobing, membranes were stripped according to the manufacturer's protocol using boiling $0.5 \%$ SDS. $\mathrm{HO}-1$ and $\beta$-actin
mRNA quantification was performed by densitometry (PDI, Santa Clara, CA, U.S.A.). The ratio of HO- 1 to $\beta$-actin was calculated.

\section{Preparation of Lung Nuclear Protein}

Rat lung nuclear proteins were collected according to published methods (2). Briefly, lung tissue was homogenized on ice with a Dounce homogenizer in a buffer containing $0.5 \mathrm{M}$ sucrose, $10 \mathrm{mM} \mathrm{N}$-2-hydroxyethylpiperazine- $N$ '-2-ethanesulfonic acid (HEPES) (pH 7.9), $1.5 \mathrm{mM} \mathrm{MgCl}, 10 \mathrm{mM} \mathrm{KCl}$, $10 \%$ glycerol, $1 \mathrm{mM}$ EDTA, and $1 \mathrm{mM}$ phenylmethyl sulfonyl fluoride (PMSF). The homogenate was centrifuged at $4000 \times$ $g$ for $20 \mathrm{~min}$ at $4^{\circ} \mathrm{C}$, and the pellet was resuspended in a high salt buffer containing $20 \mathrm{mM}$ HEPES (pH 7.9), 25\% glycerol, $0.5 \mathrm{M} \mathrm{KCl}, 1.5 \mathrm{mM} \mathrm{MgCl} 2,0.4 \mathrm{mM}$ EDTA, and $0.5 \mathrm{mM}$ PMSF. After incubation on ice for $10 \mathrm{~min}$, the supernatant was centrifuged at $14,000 \times g$ for $15 \mathrm{~min}$ at $4^{\circ} \mathrm{C}$. The supernatant was aliquoted and stored at $-80^{\circ} \mathrm{C}$. Before assays, nuclear protein content was determined by the method of Bradford (19) (Bio-Rad, Richmond, CA, U.S.A.).

\section{Determination of the AP-1 Binding Ability of Lung} Nuclear Proteins by Electrophoretic Mobility Shift Assay

A ${ }^{32}$ P-labeled oligonucleotide with the AP-1 consensus sequence (5'- CTAGTGATGAGTCAGCCGGATC-3') (Operon Technologies, Alameda, CA, U.S.A.), containing the core AP-1 binding site TGAGTCA seen in mouse HO-1 (12), was used as a probe to evaluate the AP-1 binding ability of lung nuclear protein. Nuclear proteins $(20 \mu \mathrm{g})$ were mixed with the radiolabeled AP-1 probe in a buffer containing $10 \mathrm{mM}$ HEPES (pH 7.9), $1 \mathrm{mM}$ EDTA, $80 \mathrm{mM} \mathrm{KCl}, 1 \mu \mathrm{g}$ poly[dIdC]poly[dIdC], and 4\% Ficoll. The reaction mixture was incubated at room temperature for $30 \mathrm{~min}$ and electrophoresed on $6 \%$ polyacrylamide gels. To distinguish nonspecific binding of the nuclear proteins, competition reactions were performed by adding 50-fold excess nonradiolabeled AP-1 and 100-fold mutated AP-1 (5'-CTAGTGAACTCAGTGCCCGATC-3') (Operon Technologies) to the binding reaction mixture before electrophoresis.

\section{Determination TGSH and GSSG Levels}

Lungs were washed with cold $\left(4^{\circ} \mathrm{C}\right)$ saline, ground with a cold mortar and pestle, and frozen at $-80^{\circ} \mathrm{C}$. Fifty milligram aliquots of frozen lung tissue were quickly weighed and dissolved in $50 \mu \mathrm{L}$ of $10 \%$ perchloric acid $\left(\mathrm{HClO}_{4}\right)$ to prevent GSH oxidation. In another experiment, the same amount of frozen lung tissue was homogenated in $50 \mu \mathrm{L}$ phosphate buffer to determine the protein concentration. After deproteination with $50 \mu \mathrm{L}$ of $10 \%$ metaphosphoric acid, the tissue was sonicated and centrifuged and the supernatant was diluted accordingly for TGSH and GSSG measurements. The TGSH and GSSG were determined separately using the Glutathione Assay Kit (Cayman Chemical, Ann Arbor, MI, U.S.A.) following the manufacturer's protocol. This assay kit used a carefully optimized enzymatic recycling method, using GSH reductase for the quantification of lung GSH content. There- 
fore, the TGSH was equivalent to the sum of GSH and GSSG. Because the cycling assay measured the rate of production of GSH from GSSG in the presence of glutathione reductase, the result reflected the sulfhydryls in the acid-soluble GSH. In another experiment, GSH was masked by 2-vinylpyridine for $1 \mathrm{~h}$ before the assay to determine the GSSG levels in the tissue samples. The samples were read at $405 \mathrm{~nm}$ using a microtiter plate at 5-min intervals for $30 \mathrm{~min}$. The TGSH and GSSG were determined by comparison with standards and normalized to protein content. The GSH content was obtained by subtracting the GSSG from the TGSH and the ratio of GSSG to GSH was expressed as a ratio of GSSG/GSH. The limits of detection for the TGSH and GSSH content were 0.5 and $0.2 \mu \mathrm{M}$, respectively.

\section{Statistical Analysis}

For comparison between neonatal and adult animals exposed to air or hyperoxia, the null hypothesis that there was no difference between group means was tested by a two-way factor ANOVA for multiple groups to allow for detection of age and hyperoxia effect and a relationship between the two variables. For comparison between treatment groups (saline-, diamide-, BSO-, and selenium-treated neonates exposed to hyperoxia), a one-way ANOVA was used (Statview 4.02, Abacus Concepts, Berkeley, CA, U.S.A.). Statistical significance $(p<0.05)$ between and within groups was determined by means of the Fisher method of multiple comparisons.

\section{RESULTS}

\section{Appearance of Lungs after Hyperoxic Exposure}

After hyperoxic exposure, we did not detect any visible differences in the degree of hemorrhage in the lungs of neonates compared with adults.

Lung TGSH level and GSSG/GSH ratio in the rats exposed to hyperoxia. Others have demonstrated that GSH depletion was associated with HO-1 induction in the brain $(10,18)$. To evaluate whether cellular GSH content was a factor in hyperoxia-induced HO-1 expression in this model, lung TGSH and GSSG levels were measured and the ratio of GSSG/GSH was calculated as a marker of cellular GSH content. Age was a significant factor for lung GSSG content but exposure to hyperoxia was not. Neonatal pups exposed to hyperoxia had significantly decreased GSSG compared with similarly exposed adult rats (Table 1). As to the GSH content, this increased with hyperoxia in both neonates and adults, as previously shown (20), suggesting compensatory up-regulation in hyperoxia. This was significantly affected by exposure to hyperoxia in both adults and neonates but age did not significantly modify the GSH content. TGSH content was significantly altered by exposure to hyperoxia in the adults only. Nonetheless, the changes in GSH and TGSH contents due to hyperoxia were modified by age group. For example, the neonates exposed to hyperoxia had a lesser increase in TGSH and GSH contents compared with the adults similarly exposed (Table 1.) In contrast, GSSG/GSH ratios were related to age and exposure to hyperoxia. Air-exposed adult lungs had significantly increased GSSG/GSH ratios compared with similarly exposed neonatal rats and hyperoxia significantly increased the GSSG/GSH ratio in the neonates but decreased this in the adults. (Table 1).

In vitro susceptibility of AP-1 binding to redox modification in the neonatal and adult rat lungs. Because AP-1 binding is important in hyperoxia-mediated HO-1 induction in adults but not in neonates $(2,15)$, we wanted to verify whether the neonates had less susceptibility to modification of AP-1 by oxidants or reductants. Nuclear proteins from the hyperoxiaexposed D0 and adult rats were subjected to thiol modification and evaluated for AP-1 binding in vitro. In adults and neonates, hyperoxia-mediated lung AP-1 binding was equally enhanced with the thiol-reducing agents DTT and $\beta$-mercaptoethanol, whereas the thiol-oxidizing agent, diamide, reduced hyperoxiamediated AP-1 binding (Fig. 1). The diminished AP-1 binding was restored after addition of DTT (Fig. 1). Overall, these data demonstrate that there are no maturational differences in susceptibility to redox modification of AP-1 in vitro.

Effects of in vivo GSH content modification and hyperoxia on AP-1 binding and HO-1 induction in neonates. Diamide $(25 \mathrm{mg} / \mathrm{kg})$, selenium $(5 \mu \mathrm{mol} / \mathrm{kg})$, or BSO ( $3 \mathrm{mmol} / \mathrm{kg})$ was injected into the D0 rats throughout the hyperoxic exposure. After $3 \mathrm{~d}$ of hyperoxia, diamide injection significantly increased lung GSSG and GSSG/GSH ratios in D0 pups, suggesting increased GSH oxidation (Table 2). Selenium injection and hyperoxic exposure significantly increased TGSH and GSH levels in the neonatal pups compared with saline-injected, hyperoxia-exposed neonatal controls despite no change in GSSG/GSH ratio (Table 2). With BSO injection and hyperoxic exposure, no detectable TGSH and GSH levels could be measured, indicating an almost complete depletion of lung GSH content in the neonatal lungs exposed to hyperoxia (Table 2). Surprisingly, despite these alterations in GSSG/GSH ratio and GSH levels, no increased AP-1 binding was observed in either diamide-, selenium-, or BSO-injected, hyperoxia-exposed D0 rats compared with saline-injected similarly exposed controls

Table 1. Lung glutathione status in neonatal $(<12 \mathrm{~h}$ old) and adult $(>2$ mo old) rats after 72 hyperoxia exposure

\begin{tabular}{lcccl}
\hline & TGSH $(\mathrm{nmol} / \mathrm{mg})$ & GSSG $(\mathrm{nmol} / \mathrm{mg})$ & GSH $(\mathrm{nmol} / \mathrm{mg})$ & GSSG/GSH \\
\hline $\mathrm{D} 0$ air & $8.44 \pm 1.39$ & $0.170 \pm 0.103^{*}$ & $8.27 \pm 1.44$ & $0.022 \pm 0.015$ \\
$\mathrm{D} 0 \mathrm{O}_{2}$ & $12.3 \pm 1.37$ & $0.329 \pm 0.157$ & $12.0 \pm 1.40 \dagger$ & $0.028 \pm 0.013 \dagger$ \\
Adult air $_{\text {Adult } \mathrm{O}_{2}}^{4.96 \pm 0.981}$ & $17.4 \pm 2.30 \dagger \#$ & $0.538 \pm 0.029^{*}$ & $4.42 \pm 0.952$ & $0.124 \pm 0.010^{*}$ \\
\hline
\end{tabular}

* Significant effect of age $(p<0.05)$; $\dagger$ significant effect of hyperoxia $(p<0.05)$; $p<0.05$ for age $\times \mathrm{O}_{2}$.

Data are mean \pm SD from three to five experiments. The GSH values are obtained by subtracting the GSSG from the total glutathione (TGSH) and normalized to protein content in the homogenates. D0 air, neonatal rats exposed to air; $\mathrm{D} 0 \mathrm{O}_{2}$, neonatal rats exposed to hyperoxia; Adult air, adult rats exposed to air; Adult $\mathrm{O}_{2}$, adult rats exposed to $\mathrm{O}_{2}$. 


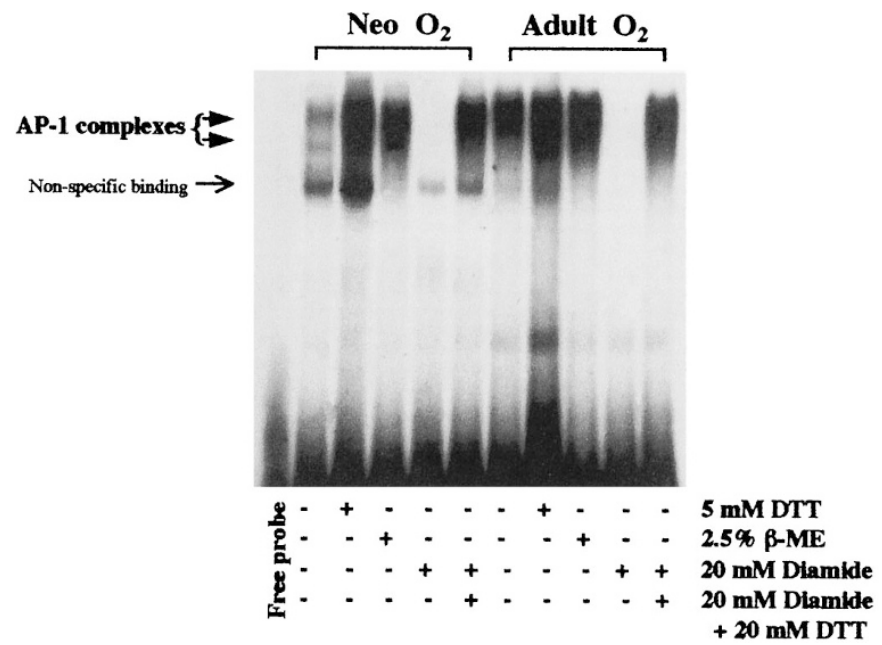

Figure 1. In vitro redox modification of lung AP-1 binding in neonatal and adult rats exposed to hyperoxia. Thiol-reducing or -oxidizing agents were incubated with lung nuclear proteins from adult or neonatal rats in the binding buffer for $20 \mathrm{~min}$ at room temperature before the addition of the ${ }^{32} \mathrm{P}$-labeled AP-1 probe. $\mathrm{Neo} \mathrm{O}_{2}$, samples collected from neonatal rat lungs exposed to hyperoxia for $72 \mathrm{~h}$; Adult $\mathrm{O}_{2}$, samples collected from adult rat lungs exposed to hyperoxia for $72 \mathrm{~h} ; D T T$, dithiothreitol; $\beta$-ME, 2-mercaptoethanol.

(Fig. 2). As to HO-1 mRNA, no change was noted after diamide injection and hyperoxia, but selenium and BSO injections, along with hyperoxic exposure, were associated with a 3and 4-fold increase in HO-1 mRNA, respectively, in the D0 rats compared with saline-injected controls similarly exposed (Fig. 3). Despite the fact that selenium and BSO caused opposite effects on the lung GSH levels, HO-1 mRNA was equally induced, suggesting that GSH levels do not modulate HO-1 mRNA in the neonatal lung.

In vivo effects of GSH content modification on hyperoxiainduced AP-1 binding and HO-1 induction in adults. To verify that diamide did not change AP-1 binding and $\mathrm{HO}-1$ mRNA in hyperoxia, diamide was also injected into adult rats twice daily before hyperoxic exposure. Surprisingly, diamideinjected adults demonstrated increased AP-1 binding and a 3-fold increase in HO-1 mRNA after hyperoxia (Figs. 4 and 5). These results suggest that in vivo GSH content modification does not result in a similar response as does in vitro manipulation. In addition, the lack of AP-1 binding and HO-1 mRNA induction in neonates after diamide injection and hyperoxia suggest that the mechanisms regulating lung AP-1 binding and HO-1 transcription in hyperoxia are different between adults and neonates.

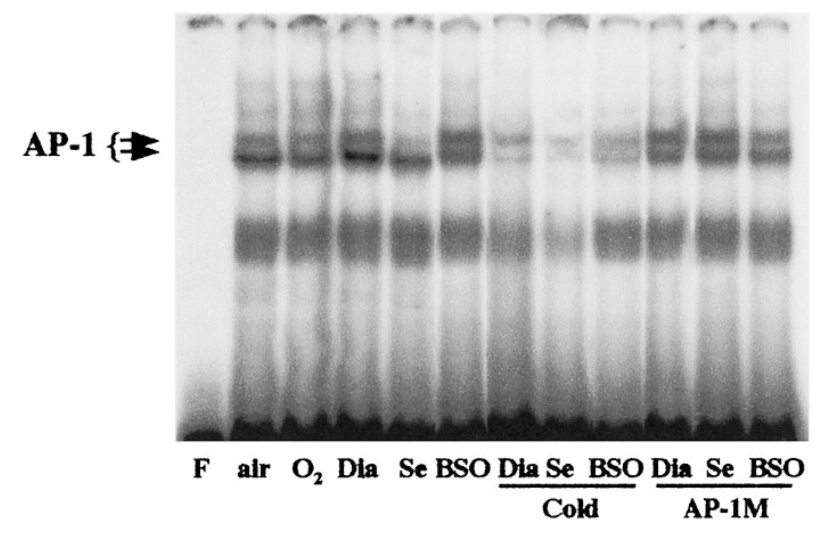

Figure 2. Lung AP-1 binding in hyperoxic neonatal rats injected with GSH-modifying agents. Lung nuclear proteins were collected after 72-h hyperoxic exposure and AP-1 binding was determined by electrophoretic mobility shift assay. The blot represents three individual experiments. $F$, free probe; air, air exposure; $O_{2}$, hyperoxia exposure with saline injection as control vehicle; Dia, injection with diamide; $S e$, injection with selenium; $B S O$, injection with BSO; Cold, cold competition with $50 \times$ unlabeled AP-1 probe; $A P-1 M$, cold competition with $100 \times$ mutant AP-1 probe.

\section{DISCUSSION}

We have previously shown that newborn animals do not increase HO-1 mRNA in response to hyperoxia whereas adults do (1). In addition, HO-1 gene expression is associated with AP-1 transactivation because several stress response elements on the HO-1 promoter are AP-1 encoded $(11,13)$. It is known that increased AP-1 binding in hyperoxia is associated with HO-1 induction in adult rat lung (15), but this is not the case in neonates (2). AP-1 transcriptional activity is thought to be regulated, at least in part, by a redox mechanism in vitro, because DNA-binding of the fos-jun heterodimer is modulated by the redox state of a single conserved cysteine residue in the DNA-binding domains of the two proteins (4). Similar mechanisms may be involved in intact cells $(4,21-23)$. Our in vitro experiments showed that both neonatal and adult lung AP-1 DNA binding in hyperoxia are equally susceptible to thiol modification, in that the thiol oxidizing agent diamide diminished AP-1 binding. However, in vivo, diamide injection did not change neonatal lung AP-1 binding in hyperoxia but further increased it in adults. Incubation with diamide also enhanced AP-1 binding in guinea pig gastric epithelial cells, and GSH content depletion inhibited the diamide-enhanced AP-1 binding (24). In these experiments, we did not observe GSH content depletion, but rather increased GSSG content and decreased

Table 2. Effect of various oxidants and reductants on neonatal lung $(<12 \mathrm{~h}$ old $)$ glutathione status after hyperoxic exposure

\begin{tabular}{lcccc}
\hline & TGSH $(\mathrm{nmol} / \mathrm{mg})$ & GSSG $(\mathrm{nmol} / \mathrm{mg})$ & GSH $(\mathrm{nmol} / \mathrm{mg})$ & GSSG/GSH \\
\hline Air & $8.54 \pm 1.39$ & $0.220 \pm 0.119$ & $8.32 \pm 1.41$ & $0.028 \pm 0.016$ \\
$\mathrm{O}_{2}$ (saline) & $10.1 \pm 2.53$ & $0.370 \pm 0.127$ & $9.78 \pm 2.57$ & $0.041 \pm 0.019$ \\
Diamide & $8.26 \pm 1.26$ & $0.620 \pm 0.302^{*}$ & $7.64 \pm 0.962$ & $0.079 \pm 0.032^{*} \dagger$ \\
Selenium & $12.4 \pm 0.86^{*}$ & $0.449 \pm 0.193$ & $12.0 \pm 0.953^{*} \dagger$ & $0.038 \pm 0.017$ \\
BSO & N/D & N/D & N/A & N/A \\
\hline
\end{tabular}

$* p<0.05 v s$ air exposed neonatal rats; $\dagger p<0.05 v s$ saline-injected neonatal rats in hyperoxia.

Data are mean \pm SD from three to five experiments. Diamide, selenium, and BSO were injected throughout the 72-h hyperoxic exposure.

Air, air exposure; $\mathrm{O}_{2}$ (saline), hyperoxic exposure with saline injection as control vehicle; Diamide, injection with diamide; Selenium, injection with selenium; $\mathrm{BSO}$, injection with 1-buthionine-[S,R] sulfoximine; N/D, nondetectable; N/A, nonapplicable. 

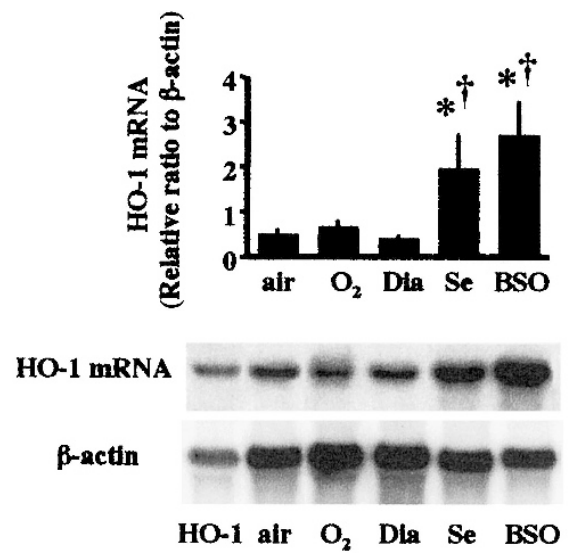

Figure 3. Lung HO-1 mRNA levels in hyperoxic neonatal rats injected with redox-modifying agents. Lung total RNA was collected after $72 \mathrm{~h}$ hyperoxic exposure and subjected to Northern analysis as described in "Methods." Upper panel: densitometric quantitation of lung HO-1 to $\beta$-actin mRNA. Values are expressed as mean $\pm \mathrm{SE}, n=3-5$ in each group. ${ }^{*} p<0.05 v s$ air exposed; $\dagger p<0.05$ vs saline injected controls. Middle panel: HO-1 mRNA. Lower panel: $\beta$-actin mRNA. HO-1, HO-1-positive control; air, air exposure; $\mathrm{O}_{2}$, hyperoxia exposure with saline injection as control vehicle; Dia, injection with diamide; $\mathrm{Se}$, injection with selenium; $B S O$, injection with BSO.

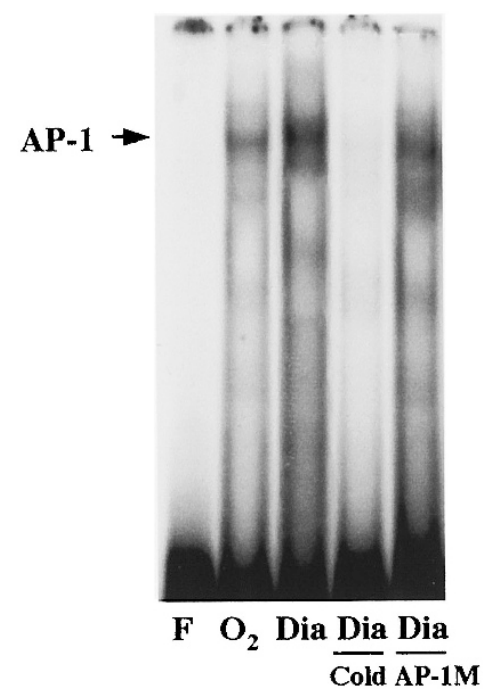

Figure 4. Lung AP-1 binding in hyperoxic adult rats with diamide injection. Lung nuclear proteins were collected after $72 \mathrm{~h}$ hyperoxia exposure and AP-1 binding was determined by electrophoretic mobility shift assay. $F$, free probe; $\mathrm{O}_{2}$, hyperoxia exposure with saline injection as control vehicle; Dia, diamide injection; cold, cold competition with $50 \times$ unlabeled AP-1 probe; $A P-1 M$, cold competition with $100 \times$ mutant AP- 1 .

GSH levels with diamide. Therefore, the increased ratio of GSSG/GSH, along with the lack of AP-1 binding and no increased HO-1 mRNA in neonates exposed to hyperoxia and injected with diamide, suggests that neonatal lung AP-1 binding and subsequent HO-1 mRNA expression in hyperoxia could be inhibited by increased GSSG/GSH ratio.

In contrast, the adult rat lung had decreased GSSG/GSH ratio and enhanced AP-1 binding activity after hyperoxic exposure. This is in agreement with the in vitro result demonstrating that AP-1 binding is favored by a more reduced thiol environment. However, adult lung AP-1 binding in hyperoxia was further enhanced by diamide injection and this was in turn
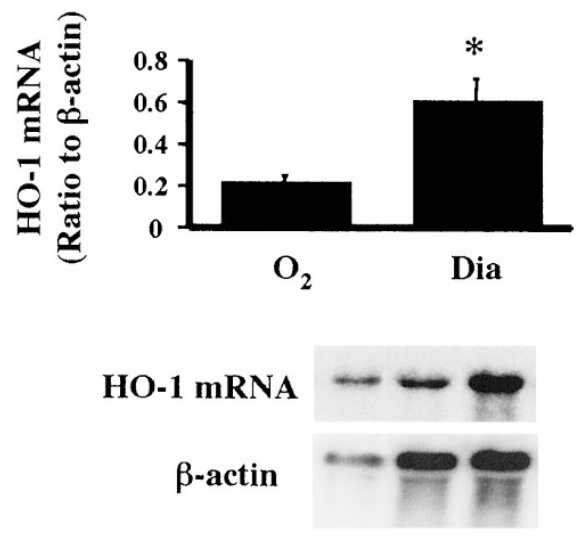

HO-1 $\mathrm{O}_{2}$ Dia

Figure 5. Northern analysis of HO-1 mRNA levels in the hyperoxic adult rat lungs. Upper panel: densitometric quantitation of lung HO-1 to $\beta$-actin mRNA. Values are expressed as mean $\pm \mathrm{SE}, n=3-5$ in each group. ${ }^{*} p<0.05$ vs saline injected controls. Middle panel: HO-1 mRNA. Lower panel: $\beta$-actin mRNA. HO-1, HO-1 positive control; $O_{2}$, hyperoxia exposure with saline injection as control vehicle; Dia, injection with diamide.

associated with increased lung HO-1 mRNA induction. This is in agreement with a previous report (16), but does not correlate with our results where diamide is added to lung nuclear extracts. Why this occurs is still unclear.

In adults, we observed lower lung GSSG/GSH ratio after hyperoxia. This is in contrast to many reports, but there is an example where, after an initial rise, the lung GSSG/GSH ratio decreased after prolonged hyperoxia (54 h) (25). Because our exposures were longer than in other studies where GSH content or GSSG/GSH ratios were measured (72 h versus $2-54 \mathrm{~h}$ ) (25), this could be an important factor in the decreased lung GSSG/ GSH ratio we observed. Another consideration is that the method we used for GSH content measurement could have also artificially increased GSSG in air exposed adults because the vinylpyridine may cause higher value of GSSG (26). We would have expected this to affect all samples if this were the case. However, this was only seen in adults exposed to air. Additionally, adults were killed by $\mathrm{CO}_{2}$ narcosis, which could have increased GSSG due to the longer time to death than that in neonates. However, we limited the exposure to $\mathrm{CO}_{2}$ to $<30$ $\mathrm{s}$ and completed the killing with decapitation, thereby limiting the time to death in the adults. Another consideration is the contribution of blood in the lungs to the GSH content of tissues. We did not see visible differences in the degree of hemorrhage in the lungs of neonates compared with adults after hyperoxia, suggesting that this is not a significant contributing factor. Nonetheless, heme content of tissues was not measured.

Neonatal AP-1 binding in hyperoxia was not altered by either diamide, selenium, or BSO, whereas HO-1 mRNA was, suggesting that transcription factors other than AP-1 may dictate the regulation of downstream genes such as HO-1 in neonates, as shown by others using cell models $(27,28)$.

In summary, in vivo changes in GSSG/GSH ratio did not similarly affect AP-1 binding and downstream gene (HO-1) expression in hyperoxia-exposed adults as it did in neonates. This is in opposition to what would have been predicted by in 
vitro results. Also, AP-1 binding occurred readily in adult lungs after hyperoxic induction, whereas neonatal lungs were relatively resistant to AP-1 binding either in hyperoxia alone or after modifications of GSH level status and exposure to hyperoxia in vivo. Nevertheless, neonatal lung HO-1 mRNA upregulation was observed with GSH content depletion, suggesting an alternative pathway of hyperoxic HO-1 regulation in neonates. It will be important to evaluate other upstream events that are modulated by hyperoxia in the neonates. Because HO-1 may mediate protection in hyperoxia at moderate levels (29), it remains to be determined whether these maturational alterations in HO-1 regulation are important in mediating tolerance to hyperoxia.

\section{REFERENCES}

1. Dennery PA, Rodgers PA, Lum MA, Jennings BC, Shokoohi V 1996 Hyperoxic regulation of lung heme oxygenase in neonatal rats. Pediatr Res 40:815-821

2. Yang G, Madan A, Dennery PA 2000 Maturational differences in hyperoxic AP-1 activation in rat lung. Am J Physiol Lung Cell Mol Physiol 278:L393-L398

3. Xanthoudakis S, Curran T 1996 Redox regulation of AP-1: a link between transcription factor signaling and DNA repair. Adv Exp Med Biol 387:69-75

4. Abate C, Patel L, Rauscher FJD, Curran T 1990 Redox regulation of fos and jun DNA-binding activity in vitro. Science 249:1157-1161

5. Meyer M, Pahl HL, Baeuerle PA 1994 Regulation of the transcription factors NF-kappa B and AP-1 by redox changes. Chem Biol Interact 91:91-100

6. Xia C, Hu J, Ketterer B, Taylor JB 1996 The organization of the human GSTP1-1 gene promoter and its response to retinoic acid and cellular redox status. Biochem J 313:155-161

7. Schenk H, Klein M, Erdbrügger W, Dröge W, Schulze-Osthoff K 1994 Distinct effects of thioredoxin and antioxidants on the activation of transcription factors NF-kappa B and AP-1. Proc Natl Acad Sci USA 91:1672-1676

8. Schulze-Osthoff K, Los M, Baeuerle PA 1995 Redox signalling by transcription factors NF-kappa B and AP-1 in lymphocytes. Biochem Pharmacol 50:735-741

9. Hirota K, Matsui M, Iwata S, Nishiyama A, Mori K, Yodoi J 1997 AP-1 transcriptional activity is regulated by a direct association between thioredoxin and Ref- 1 . Proc Natl Acad Sci USA 94:3633-3638

10. Ewing JF, Maines MD 1993 Glutathione depletion induces heme oxygenase-1 (HSP32) mRNA and protein in rat brain. J Neurochem 60:1512-1519

11. Alam J 1994 Multiple elements within the $5^{\prime}$ distal enhancer of the mouse heme oxygenase-1 gene mediate induction by heavy metals. J Biol Chem 269:25049-25056

12. Alam J, Cai J, Smith A 1994 Isolation and characterization of the mouse heme oxygenase-1 gene. Distal 5' sequences are required for induction by heme or heavy metals. J Biol Chem 269:1001-1009
13. Alam J, Camhi S, Choi AM 1995 Identification of a second region upstream of the mouse heme oxygenase-1 gene that functions as a basal level and inducer-dependent transcription enhancer. J Biol Chem 270:11977-11984

14. Alam J, Den Z 1992 Distal AP-1 binding sites mediate basal level enhancement and TPA induction of the mouse heme oxygenase-1 gene. J Biol Chem 267:21894-21900

15. Lee PJ, Alam J, Sylvester SL, Inamdar N, Otterbein L, Choi AM 1996 Regulation of heme oxygenase-1 expression in vivo and in vitro in hyperoxic lung injury. Am J Respir Cell Mol Biol 14:556-568

16. Tomaro ML, Frydman J, Frydman RB 1991 Heme oxygenase induction by $\mathrm{CoCl}_{2}$, Co-protoporphyrin IX, phenylhydrazine, and diamide: evidence for oxidative stress involvement. Arch Biochem Biophys 286:610-617

17. Othman AI, El Missiry MA 1998 Role of selenium against lead toxicity in male rats. J Biochem Mol Toxicol 12:345-349

18. Oguro T, Hayashi M, Numazawa S, Asakawa K, Yoshida T 1996 Heme oxygenase-1 gene expression by a glutathione depletor, phorone, mediated through AP-1 activation in rats. Biochem Biophys Res Commun 221:259-265

19. Bradford MM 1976 A rapid and sensitive method for the quantitation of microgram quantities of protein utilizing the principle of protein-dye binding. Anal Biochem $72: 248-254$

20. Kennedy KA, Lane NL 1994 Effect of in vivo hyperoxia on the glutathione system in neonatal rat lung. Exp Lung Res 20:73-83

21. Xanthoudakis S, Miao G, Wang F, Pan YC, Curran T 1992 Redox activation of Fos-Jun DNA binding activity is mediated by a DNA repair enzyme. EMBO J 11:3323-3335

22. Xanthoudakis S, Curran T 1992 Identification and characterization of Ref-1, a nuclear protein that facilitates AP-1 DNA-binding activity. EMBO J 11:653-665

23. Okamoto T, Ogiwara H, Hayashi T, Mitsui A, Kawabe T, Yodoi J 1992 Human thioredoxin/adult $\mathrm{T}$ cell leukemia-derived factor activates the enhancer binding protein of human immunodeficiency virus type 1 by thiol redox control mechanism. Int Immunol 4:811-819

24. Rokutan K, Teshima S, Miyoshi M, Kawai T, Nikawa T, Kishi K 1998 Glutathione depletion inhibits oxidant-induced activation of nuclear factor-kappa B, AP-1, and c-Jun/ATF-2 in cultured guinea-pig gastric epithelial cells. J Gastroenterol 33:646655

25. Beehler CJ, Simchuk ML, Toth KM, Drake SK, Parker NB, White CW, Berger EM, Sanderson RJ, Repine JE 1989 Blood sulfhydryl level increases during hyperoxia: a marker of oxidant lung injury. J Appl Physiol 67:1070-1075

26. Schofield D, Mei G, Braganza JM 1993 Some pitfalls in the measurement of blood glutathione. Clin Sci (Colch) 85:213-218

27. Alam J, Stewart D, Touchard C, Boinapally S, Choi AM, Cook JL 1999 Nrf2, a Cap'n'Collar transcription factor, regulates induction of the heme oxygenase-1 gene. J Biol Chem 274:26071-26078

28. Alam J, Wicks C, Stewart D, Gong P, Touchard C, Otterbein S, Choi AM, Burow ME, Tou J 2000 Mechanism of heme oxygenase-1 gene activation by cadmium in MCF-7 mammary epithelial cells. Role of p38 kinase and Nrf2 transcription factor. J Biol Chem 275:27694-27702

29. Suttner DM, Dennery PA 1999 Reversal of HO-1 related cytoprotection with increased expression is due to reactive iron. FASEB J 13:1800-1809 